\title{
Propiedades físicas del suelo en diferentes sistemas agrícolas en la provincia de Los Ríos, Ecuador
}

\author{
Advantages of incorporating new varieties in the cultivation \\ of cotton in the Sinú Valley
}

\author{
Indira Dayanara Novillo Espinoza ${ }^{1}$, Manuel Danilo Carrillo Zenteno ${ }^{2 *}$, Jessica Elizabeth Cargua Chavez ${ }^{3}$, \\ Virginia Nabel Moreira ${ }^{4}$, Karla Estefania Albán Solarte ${ }^{5}$, Fátima Lourdes Morales Intriago ${ }^{6}$.
}

Recibido para publicación: Octubre 27 de 2017 - Aceptado para publicación: Mayo 24 de 2018

\begin{abstract}
RESUMEN
Los sistemas de monocultivo degradan el suelo afectando sus propiedades físicas y químicas, y su regeneración es muy lenta. Con el objetivo de conocer cambios en las propiedades físicas del suelo de la Estación Experimental Tropical Pichilingue, por efecto del uso, se empleó un diseño de bloques completos al azar con tres repeticiones, en los suelos de bosque nativo y monocultivos de maíz (30 años), cacao (50 años), pasto (4 años) y palma aceitera (26 años) en diferentes profundidades cada 0,10 m hasta los 0,6 m. Se evaluaron propiedades físicas como conductividad hidráulica, densidad aparente, densidad real, porosidad total, porosidad de aireación, humedad volumétrica, textura, arcilla dispersa en agua, grado de floculación, materia orgánica e índice de materia orgánica/ limo+arcilla. Los datos fueron analizados estadísticamente y se realizó la prueba de Tuckey $(\mathrm{P} \leq 0,05)$. Se encontró que maíz, palma aceitera y pasto provocaron incrementos estadísticos significativos en la densidad aparente del suelo sin llegar a sobrepasar los niveles críticos de 1390 kg m-3 y no significativos en reducción de la porosidad total en la profundidad de 0,1-0,2 m. También, hubo alta concentración de arcillas en las primeras profundidades de los suelos cultivados, que provocó susceptibilidad a los procesos de erosión hídrica, concluyendo que el suelo bajo cultivo de palma aceitera mostró mayores diferencias estadísticas debido a la textura, afectando negativamente la densidad aparente del suelo, conductividad hidráulica, arcilla dispersa en agua, que en suma contribuyen a disminuir la estabilidad de agregados.
\end{abstract}

Palabras clave: Arcilla dispersa en agua, grado de floculación, índice de materia orgánica, erosión, usos de suelo.

\begin{abstract}
Monoculture systems degrade the soil, affecting its physical and chemical properties, and its regeneration is very slow. With the objective of knowing changes in the physical properties of the Pichilingue Tropical Experimental Station, due to the use effect, a randomized complete block design with three replications was used in native forest soils and corn monocultures (30 years), cacao (50 years), pasture ( 4 years) and oil palm (26 years) at different depths every $0.10 \mathrm{~m}$ to $0.6 \mathrm{~m}$. were evaluated physical properties such as hydraulic conductivity, bulk density, real density, total porosity, aeration porosity, volumetric moisture, texture, clay dispersed in water, degree of flocculation, organic matter and organic matter / silt + clay index, The data were statistically analyzed and Separation test Tukey $(P \leq 0.05)$. It was found that corn, oil palm and grass caused significant statistical increases in the apparent density of the soil without exceeding the critical levels of $1390 \mathrm{~kg} \mathrm{~m}-3$ and not significant in reducing the total porosity in the depth of 0.1-0.2 m. Also, there was a high concentration of clays in the first depths of the soils under monoculture that caused susceptibility to water erosion processes, concluding that the soil under oil palm cultivation showed greater statistical differences due to the texture, negatively affecting the apparent density of the soil, hydraulic conductivity, clay dispersed in water, which in sum contribute to decrease the stability of aggregates.
\end{abstract}

Key words: clay dispersed in water, degree of flocculation, organic matter index, erosion, soil uses.

\footnotetext{
${ }^{1}$ Ingeniera Ambiental, Universidad Tecnológica Equinoccial Sede Santo Domingo (UTE-SD), Santo Domingo de los Tsáchilas, Ecuador. ${ }^{2 *} \mathrm{PhD}$. Profesor de la UTE-SD, Departamento de Manejo de Suelos y Aguas (DMSA) de la Estación Experimental Tropical Pichilingue (EETP) del Instituto Nacional de Investigaciones Agropecuarias (INIAP). Quevedo, Los Ríos, Ecuador. ORCID 0000-0001-7062-8248. Celular 593 969353137. manuel.carrillo@iniap.gob.ec.

${ }^{3}$ Ingeniero Agropecuario. Departamento de Manejo de Suelos y Aguas DMSA Instituto Nacional de Investigaciones Agropecuarias INIAP. Quevedo, Los Ríos, Ecuador.

${ }^{4}$ Ingeniera Agropecuario. Departamento de Manejo de Suelos y Aguas DMSA Instituto Nacional de Investigaciones Agropecuarias INIAP. Quevedo, Los Ríos, Ecuador.

${ }^{5}$ Ingeniera Ambiental, UTE-SD, Santo Domingo de los Tsáchilas, Ecuador.

${ }^{6}$ M.Sc. Extensión Rural. Profesor. Universidad Técnica de Babahoyo Extensión-Quevedo, Quevedo, Los Ríos, Ecuador
} 


\section{INTRODUCCIÓN}

El suelo es un recurso natural no renovable y su regeneración es muy lenta, siendo sometido constantemente a los procesos de destrucción y degradación. Es un elemento fundamental para la agricultura por proveer de agua y nutrientes a los cultivos; además, interviene en los ciclos del agua, carbono, nitrógeno, fósforo y otros elementos de interés (Ferreras et al., 2015). La industrialización, globalización y el crecimiento demográfico ha ocasionado la expansión de la frontera agrícola, utilizándose más espacio de suelo para dicha actividad, provocando deterioro en sus propiedades físicas, químicas y biológicas naturales, conduciendo a una degradación paulatina (FAO, 2016). Los cambios edáficos por el uso del suelo, no sólo surgen por la expansión agrícola o modificaciones en el sistema de labranza utilizado, la secuencia y rendimiento de los cultivos implantados, también pueden afectar (Díaz-Zorita y Álvarez, 2004); así, los cultivos intensivosdegradanelsuelodebidoalareducción y/o eliminación de su cobertura, estabilidad de agregados, cantidad de materia orgánica; además promueven la erosión, compactación y reduce la productividad (Toledo et al., 2013).

Para la FAO-UNESCO, la degradación del suelo es un proceso que disminuye la capacidad actual y potencial del suelo para producir cuantitativa y cualitativamente, bienes y servicios, por acciones directas de origen antrópicas relacionadas con el manejo de agroquímicos y riego en la agricultura, forestería, ganadería, y por acciones indirectas relacionadas con actividades industriales, eliminación de residuos y transporte entre otras (Brissio y Savini, 2005). Se reconocen tres tipos de degradación del suelo: física, química y biológica (FAO, 2011). La degradación física del suelo inhabilita el funcionamiento adecuado del suelo, debido a que afecta su capacidad de transmisión de fluidos, el volumen de almacenaje relacionado con el balance de gases y agua necesario para disolver los nutrientes para las plantas (Muñoz et al., 2013). La pérdida de la calidad física de un suelo, puede también ser evaluada por la alteraciónen la densidad, porosidad, distribución del tamaño de poros, estructura y la tasa de infiltración de agua en el suelo (FAO, 2000).

La conductividad hidráulica (Ch) es un parámetro que indica la facilidad con la que los poros del suelo permiten el movimiento del agua y es dependiente de la porosidad, permeabilidad y grado de saturación presente (Barbecho y Calle, 2012), teniendo relación directamente proporcional con la porosidad (Bastos y Oliveira, 2004). La densidad aparente del suelo (Da) que es la relación entre el volumen total de sólidos del suelo y su masa (Alvarado y Forsythe, 2011), utilizada como indicador de calidad del suelo, con la que se puede determinar alteraciones producidas por las actividades antrópicas como uso de arado, maquinaria pesada, cultivos, pudiendo indicar el grado de compactación del suelo y las limitaciones para el crecimiento de las raíces (Miralles, 2006). La densidad real (Dr) es generalmente constante debido a que es determinada por la mineralogía y composición química de la fase sólida del suelo (Ramírez et al., 2015).

Laporosidad total (Pt) representa el porcentajedel volumen de suelo que no se encuentra ocupado por sólidos y está constituido aproximadamente del $50 \%$ por materiales sólidos y el otro $50 \%$ por espacio poroso, donde nutrientes, aire, gases y agua pueden circular (FAO, 2016). La porosidad de aireación es la que no retiene agua y sirve para el intercambio gaseoso, por tanto, genera una idea de la proporción de poros de mayor tamaño (FAO, 2016). La textura se refiere a la proporción de los componentes inorgánicos del suelo, arena, limo y arcilla. Esta propiedad influye en la fertilidad y la capacidad de retención de agua, aireación y contenido de materia orgánica (FAO, 2016). Un alto porcentaje de arcilla dispersa en agua 
(ADA) indica que la solución del suelo es sódica, los poros del suelo se cierran y se forma una capa superficial sellada, lo cual afecta a la infiltración y aumenta el riesgo a erosión (Condé et al., 2012); además, refleja la facilidad de compactación del suelo (Santos et al., 2010). El grado de floculación (GF) o dispersión de la arcilla se ve afectado por factores como la actividad bacteriana que varía según los niveles de acidez del suelo (Lima et al., 2013), influyendo en la agregación del suelo (Santos et al., 2010). Cuanto mayor sea el GF, menor será la tendencia a la desagregación de micro agregados estabilizados por MO u óxidos de Fe y Al (Hillel, 2013).

El uso de fertilizantes orgánicos y los sistemas de preparación del suelo también provocan reducción en el grado de floculación, debido a que los compuestos orgánicos presentes, influyen en las cargas sobre las superficies coloidales (Rauber et al., 2012). La materia orgánica $(\mathrm{MO})$ se encuentra de tres formas en el suelo, cómo una composición próxima al carbono elemental, como humus y humatos, y; como residuos orgánicos poco alterados (Alonso et al., 2007), conocida también según Loss et al. (2014) como materia orgánica lábil (MOL), siendo todas ellas consideradas como un indicador de la calidad del suelo, ya que ejerce un efecto positivo en sus propiedades físicas (Eyherabide et all., 2014).

El Índice de $\mathrm{MO}(\mathrm{MO} / \mathrm{Li}+\mathrm{Arc}, \mathrm{IMO})$ expresa la susceptibilidad del suelo a la degradación, siendo un indicador más sensible que la $\mathrm{MO}$ con respecto al estado del suelo, siendo recomendable su uso cuando los suelos analizados, difieren en su granulometría (Quiroga et al., 2006). De esta forma, valores de IMO menores a cinco, prueban la existencia de problemas en el manejo de suelo debido a que señalan existencia de bajo contenido de MO (Vankeirsbilck, 2014); en tanto que, la relación de IMO mayor de cinco, es considerado como el valor con el que los suelos tienen alto riesgo de degradación (Pieri, 1995, citado por Quiroga et al., 2006).

En la agricultura ecuatoriana se ha usado un enfoque agronómico más que ecológico, propiciando el aumento de la producción con la apertura de nuevas áreas de cultivo y promoviendo el aumento de la productividad mediante la utilización de tecnología por la denominada revolución verde (Suquilanda, 2008), que ha afectado directamente el ecosistema natural de los suelos. Basado en los antecedentes, la presente investigación planteó como objetivo determinar los cambios en las propiedades físicas del suelo de la Estación Experimental Tropical Pichilingue (EETP) por efecto del uso como monocultivo, para generar conocimiento y dar un manejo adecuado a este recurso.

\section{MATERIALES Y MÉTODOS}

Esta investigación fue ejecutada en el primer semestre del 2017, en los predios de la Estación Experimental Tropical Pichilingue (EETP) del Instituto Nacional de Investigaciones Agropecuarias (INIAP), ubicada en el km 5 vía Quevedo - El Empalme, cantón Mocache, Provincia de Los Ríos, Ecuador. Geográficamente localizada en las Coordenadas $01^{\circ} 06^{\prime}$ Latitud Sur y $79^{\circ} 28^{\prime}$ Longitud Oeste; con una altitud de 120 msnm. Los suelos de esta zona se clasifican como Fluvisoles éutricos, tendiendo a la acidez por presentar una saturación de bases del 50\%; además, se inundan de manera natural por encontrarse en depósitos aluviales y presentan gran cantidad de humus en la capa superficial del suelo (Gardi et al., 2014).

Fueron evaluados cinco sistemas de uso de suelo de la EETP, bosque primario (guayacán, Tabebuia chrysantha Jacq; guarumo, Cecropia peltata; heliconias, Heliconia sp.; ortiga, Urtica dioica; y pachaco, Schizolobium parahybum), maíz (Zea mays L.) por 30 años, cacao 
(Theobroma cacao L.) por 50 años, pasto guinea (Panicum maximun Jacq.) por 4 años y palma aceitera (Elaeis guineensis Jacq) por 26 años. En cada sistema se colectaron seis muestras que corresponden a las profundidades de muestreo $0,0-0,1 \mathrm{~m} ; 0,1-0,2 \mathrm{~m} ; 0,2-0,3 \mathrm{~m} ; 0,3-0,4 \mathrm{~m} ; 0,4$ - 0,5 m y 0,5 - 0,6 m, todas replicadas tres veces, teniendo un total de 90 unidades de muestreo. Fueron evaluadas la conductividad hidráulica (Ch), usando un permeámetro de carga constante y para el cálculo se utilizó la Ley de Darcy (ecuación 1) (Klein, 2008). La densidad aparente del suelo (Da) empleando el método del terrón parafinado, dividiendo la masa del suelo seco a $105^{\circ} \mathrm{C}$, considerando el espacio poroso, para el volumen del terrón (Gabriels y Lobo, 2011). Para la densidad real (Dr) se dividió la masa del suelo seco para su volumen, sin considerar el espacio poroso, utilizando el método del balón volumétrico (Klein, 2008).

Ecuación de Darcy

$\mathrm{Ko}=\mathrm{q}(\partial \mathrm{H} / \partial \mathrm{z})$

(Ecuación

Donde: $K_{0}=$ Conductividad hidráulica; $\mathrm{q}=$ Densidad de flujo (mm h-1); $\partial \mathrm{H}=$ altura del sistema suelo + agua $(\mathrm{cm}) ; \partial z=$ altura de suelo (cm).

$$
\mathrm{q}=\mathrm{Q} / \mathrm{At}
$$

Donde: $\mathrm{q}=$ Densidad de flujo $=\mathrm{Ko}(\partial \mathrm{H} / \partial \mathrm{z})$; $\mathrm{Q}=$ Volumen de agua drenada $(\mathrm{mL} \mathrm{o} \mathrm{cm} 3) ; \mathrm{A}$ $=$ Área del cilindro ( $\pi$. r2); $\mathrm{t}=$ Tiempo (min).

Además, fueron determinadas la porosidad total del suelo $(\mathrm{Pt})$ con ayuda de la ecuación 2 y porosidad de aireación (Pa) con la ecuación 3, sustrayendo la humedad volumétrica $(\mathrm{Hv})$ de la porosidad total $(\mathrm{Pt})$;

$$
\operatorname{Pt}(\%)=(1-(\mathrm{Da} / \mathrm{DH} 2 \mathrm{O}) * 100) \quad(\text { Ecuación } 2)
$$

$\mathrm{Pa}=(\mathrm{Pt}-\mathrm{Hv})$

(Ecuación

Donde: $\mathrm{Hv}=$ humedad volumétrica $=\left(\mathrm{Hg}^{*} \mathrm{Da} /\right.$ $\left.\mathrm{D}_{\mathrm{H} 2 \mathrm{O}}\right) ; \mathrm{Da}=$ densidad aparente del suelo (t m-3); $\mathrm{D}_{\mathrm{H} 2 \mathrm{O}}=$ densidad del agua $\left(\mathrm{t} \mathrm{m}^{-3}\right)$;
$\mathrm{Hg}=$ humedad gravimétrica $=(($ Msh-Mss $)) /$ Mss; Msh y Mss = masa de suelo húmedo y suelo seco, respectivamente (Klein, 2008).

La textura fue determinada usando el método de Boyoucos o del hidrómetro (Klein, 2008); arcilla dispersa en agua (ADA) con la metodología del EMBRAPA (2011), siendo la misma utilizada para determinación de textura, exceptuando la adición de la solución dispersante. También el índice de arcilla dispersa en agua que resulta de la división entre el porcentaje de ADA y el porcentaje de arcilla total (Pragana et al., 2012). El grado de floculación (GF) determinado con la ecuación 4 indicada por (Lima et al., 2013); materia orgánica (MO) determinada con el método de Wakley-Black (EMBRAPA, 2011). El índice de MO (IMO) calculada con la relación entre el porcentaje de materia orgánica y la suma de los porcentajes de limo más arcilla y el valor resultante se multiplica por 100 para obtener un número entero (Pieri, 1995, citado por Quiroga et al., 2006).

$\mathrm{Gf}=100 *($ arcilla total-arcilla dispersa en agua $) /$ arcilla total

(Ecuación 4)

Los resultados de cada variable en los diferentes usos del suelo y profundidades, fueron analizados estadísticamente usando un Diseño de Bloques Completos al Azar, con tres repeticiones y las diferencias y similitudes entre medias de tratamientos, separadas con la Prueba de Tukey $(\mathrm{P}=\leq 0,05)$ empleando el programa estadístico INFOSTAT (Di Rienzo et al., 2011). Para mejorar el valor de CV, los datos fueron transformados usando la raíz cuadrada de $\mathrm{X}$.

\section{RESULTADOS Y DISCUSIÓN}

De las variables evaluadas la Dr, Pt, Pa, ADA y Gf, no presentaron diferencias estadísticas significativas entre coberturas de suelo y profundidad, indicativo de que estas variables no son adecuadas para determinar los efectos de estos sistemas sobre el suelo. 
La Ch presenta diferencias estadísticas significativas entre los sistemas de uso en la profundidad de 0,3-0,4 m (Tabla 1), mientras que, en profundidades mayores, no se registraron diferencias estadísticas significativas. Los coeficientes de variación fluctuaron entre 76\% y $247 \%$, debido a la alta variabilidad en las propiedades físicas que presentaron los suelos (Silva, 2003). Estos valores resultaron semejantes a los encontrados por Lozano et al. (2005), Guatibonza et al. (2009) y Abreu et al. (2004) y elevados si comparados con los reportados por Villafuerte (2016), para Santo Domingo de los Tsáchilas, en varios sistemas de usos del suelo.

En la profundidad de 0,3-0,4 m, el suelo bajo bosque mostró un promedio Ch de 0,02 mm $\mathrm{h}^{-1}$, valor que resultó estadísticamente igual a los destinados al cultivo de maíz, cacao y palma; sin embargo, fue diferente a los 1,65 $\mathrm{mm} \mathrm{h}^{-1}$ encontrado en el suelo con pastizal. Los valores de Ch en los diferentes usos de suelo, varían de 0,02 hasta $7,91 \mathrm{~mm} \mathrm{~h}^{-1}$, que para SCS-USDA (1993) los clasifica como Ch muy baja $\left(<0,036 \mathrm{~mm} \mathrm{~h}^{-1}\right)$ y moderadamente baja $\left(0,36-3,6 \mathrm{~mm} \mathrm{~h}^{-1}\right)$, siendo que Kutilek y Nielsen (1994) los denomina como Ch muy baja $\left(0,36 \mathrm{~cm} \mathrm{~h}^{-1}\right)$ y media $\left(3,6 \mathrm{~cm} \mathrm{~h}^{-1}\right)$. En la primera profundidad, 0,0-0,1 $\mathrm{m}$ de los suelos bajo bosque, cultivo de maíz, cacao y palma aceitera, los valores de Ch fueron mayores que en el resto de profundidades en las cuales tiende a disminuir a partir de la profundidad de 0,1-0,2 m; mientras que, en el cultivo de pasto, la Ch aumentó desde la profundidad de 0,1 m hasta 0,4 m y disminuyó en las siguientes; siendo efecto de la presencia de raíces fasciculadas propias de las gramíneas (Bravo et al., 2004). La Da no presentó diferencias estadísticas significativas por profundidad dentro de cada sistema de uso del suelo; sin embargo, existieron diferencias entre sistemas de uso de suelo en la profundidad de 0,0-0,1 m, siendo la Da de 834 kg m-3 encontrado en el cultivo de maíz diferente a los restantes sistemas que mostraron valores superiores a
$1050 \mathrm{~kg} \mathrm{~m}^{-3}$ (Tabla 1). Este efecto es debido a que el horizonte superficial es mecanizado frecuentemente, lo cual provocó reducción del valor de Da (Álvarez y Steinbach, 2009).

En el cultivo de maíz y pasto, fue evidente un aumento en la Da en la segunda profundidad de muestreo 0,1-0,2 m (especialmente bajo cultivo de maíz que presentó diferencias estadísticas significativas), como consecuencia del pase superficial de los aperos agrícolas y el pisoteo de ganado, respectivamente; sin embargo, estos dos valores no llegaron al valor crítico de $1600 \mathrm{~kg} \mathrm{~m}^{-3}$, según la textura (USDA, 2015), por lo cual no se consideran compactados y no afectarían el crecimiento de la raíces (Reinert et al., 2008). Igual efecto se observó en las primeras tres profundidades de los tratamientos estudiados, considerando que el suelo fue de textura franca, no sobrepasaron el valor crítico, indicando que el suelo no presentó problemas de compactación. Sin embargo, para el cultivo de palma aceitera, en las tres últimas profundidades presentó textura arcillosa y el valor de Da de $1506 \mathrm{~kg} \mathrm{~m}^{-3}$, superando el valor crítico de $1390 \mathrm{~kg} \mathrm{~m}^{-3}$ (USDA, 2015). La humedad volumétrica resultó con diferencias estadísticas significativas únicamente en el cultivo de maíz, siendo la profundidad de 0-0,1 m diferente a la profundidad 0,5-0,6 m (Tabla 1). La variación en la Hv fue desde $0,04 \mathrm{~m} 3 \mathrm{~m}-3$ hasta $0,28 \mathrm{~m}^{3} \mathrm{~m}^{-3}$, lo que indicó grandes diferencias en el agua disponible para las plantas, evidenciando que excepto en el sistema de bosque, los restantes tratamientos presentaron disminución en la acumulación del agua a partir de los 0,4 $\mathrm{m}$ de profundidad.

Los suelos de los sistemas evaluados variaron en su textura, siendo que en el cultivo de palma se encontrarondiferenciasestadísticassignificativas en profundidad (Tabla 2), presentando en los primeros $0,2 \mathrm{~m}$ contenidos de arena superiores (40 y $41 \%$ ) a las restantes profundidades cuyas proporciones no llegaron al 30\%. 
TEMAS AGRARIOS - Vol. 23:(2) Julio - Diciembre 2018 (177 - 187)

Tabla 1. Variación en la conductividad hidráulica $\left(\mathrm{mm} \mathrm{h}^{-1}\right)$, densidad aparente $\left(\mathrm{kg} \mathrm{m}^{-3}\right)$ y humedad volumétrica $\left(\mathrm{m}^{3} \mathrm{~m}^{-3}\right)$ del suelo, a seis profundidades de acuerdo a la cobertura, en la Provincia de Los Ríos Ecuador.

\begin{tabular}{|c|c|c|c|c|c|c|c|c|c|c|c|c|c|c|c|c|c|c|}
\hline \multirow{2}{*}{ Profundidad } & \multicolumn{6}{|c|}{ CONDUCTIVIDAD HIDRÁULICA } & \multicolumn{6}{|c|}{ DENSIDAD APARENTE DEL SUELO } & \multicolumn{6}{|c|}{ HUMEDAD VOLUMÉTRICA } \\
\hline & B & $\mathbf{M}$ & C & $\mathbf{P}$ & PA & $\mathrm{CV}_{1}$ & B & $M$ & C & $\mathbf{P}$ & PA & $\mathrm{CV}_{1}$ & B & $\mathbf{M}$ & C & $\mathbf{P}$ & PA & $\mathrm{CV}_{1}$ \\
\hline m & & & $\mathrm{mm} \mathrm{h}^{-1}$ & & & $\%$ & & & $\mathrm{~g} \mathrm{~g} \mathrm{~m}^{-3}$ & & & $\%$ & & & $0^{3} \mathbf{m}^{-3}$ & & & $\%$ \\
\hline $0-0,1$ & $6,52 \mathrm{~A}$ & 5,75 & 7,91 & 0,71 & 1,28 & $116 n s$ & $1073,6 a b$ & $834 \mathrm{Bb}$ & $1228 \mathrm{a}$ & $1159 a$ & $1170 \mathrm{a}$ & $10^{*}$ & 14 & $28 \mathrm{~A}$ & 18 & 24 & 13 & $35 \mathrm{~ns}$ \\
\hline $0,1-0,2$ & $0,46 \mathrm{~A}$ & 0,45 & 0,56 & 1,53 & 1,69 & $96 \mathrm{~ns}$ & 1081,0 & $1236 \mathrm{AB}$ & 1072 & 1461 & 1253 & $14 \mathrm{~ns}$ & 16 & $13 \mathrm{AB}$ & 17 & 16 & 10 & $44 \mathrm{~ns}$ \\
\hline $0,2-0,3$ & $0,05 \mathrm{~A}$ & 1,56 & 0,17 & 2,28 & 0,71 & $101 \mathrm{~ns}$ & 1243,0 & $1321 \mathrm{~A}$ & 1182 & 1189 & 1366 & $13 \mathrm{~ns}$ & 12 & D. & 17 & 17 & 16 & $43 \mathrm{~ns}$ \\
\hline $0,3-0,4$ & $0,02 \mathrm{Ab}$ & $0,79 a b$ & $0,26 a b$ & $1,65 a$ & 0,06ab & $102^{*}$ & 1121,1 & $1278 \mathrm{~A}$ & 1278 & 1153 & 1341 & $9 \mathrm{~ns}$ & 11 & $12 \mathrm{AB}$ & 15 & 18 & 15 & $39 \mathrm{~ns}$ \\
\hline $4-0,5$ & $0,56 \mathrm{~A}$ & 0,17 & 0,47 & 0,61 & 0,37 & $108 \mathrm{~ns}$ & 1140,8 & $1242 \mathrm{AB}$ & 1263 & 1183 & 1506 & $14 \mathrm{~ns}$ & 12 & $9 \mathrm{AB}$ & 10 & 10 & 4 & $55 \mathrm{~ns}$ \\
\hline $0,5-0,6$ & $0,05 \mathrm{~A}$ & 0,08 & 0,33 & 0,97 & 0,43 & $176 \mathrm{~ns}$ & 822,9 & $1251 \mathrm{AB}$ & 1248 & 1201 & 1487 & $19 \mathrm{~ns}$ & 18 & $5 B$ & 12 & 13 & 5 & $53 \mathrm{~ns}$ \\
\hline $\mathrm{CV}_{2}(\%)$ & $234 \mathrm{~ns}$ & $246 \mathrm{~ns}$ & $187 \mathrm{~ns}$ & $63 \mathrm{~ns}$ & $77 \mathrm{~ns}$ & & $19 \mathrm{~ns}$ & $13^{*}$ & $11 \mathrm{~ns}$ & $12 \mathrm{~ns}$ & $13 \mathrm{~ns}$ & & $45 \mathrm{~ns}$ & $29^{*}$ & $28 \mathrm{~ns}$ & $29 \mathrm{~ns}$ & $27 \mathrm{~ns}$ & \\
\hline
\end{tabular}

B: bosque, M: maíz, C: cacao, P: pasto, PA: palma aceitera, $\mathrm{CV}_{1}(\%)$ : Coeficiente de variación por sistemas de uso de suelo, $\mathrm{CV}_{2}(\%)$ : Coeficiente de variación por profundidades, ns: estadísticamente no significativo, *: significativo al 5\%, letras minúsculas: significancia entre sistemas de uso de suelo, letras mayúsculas: significancia entre profundidades.

Solo en el cultivo de cacao se presentó diferencias estadísticas significativas entre profundidades, en la proporción de limo. En los primeros 0,3 m seencontró el limo en proporción mayor de 40\%, valores estadísticamente diferentes a los observados en la profundidad de 0,4 a 0,6 m, con porcentaje menor de $35 \%$. Los CV de arena por profundidades fluctuaron entre $8,0 \%$ y $16,4 \%$, en limo entre $5,3 \%$ y $16,3 \%$ y; para arcilla entre $18,1 \%$ y $28,5 \%$, todos son porcentajes aceptables y que agregan confianza a los resultados.

Por efecto del sistema de uso del suelo, se observaron diferencias estadísticamente significativas en las proporciones de arena $y$ arcilla (Tabla 2). En las profundidades de 0,3-0,4 $\mathrm{m}$ y $0,5-0,6 \mathrm{~m}$ el contenido de arena fue mayor en el bosque ( $40 \%$ de arena) y estadísticamente diferente al de palma donde la cantidad de arena no sobrepasó el 25\%. En la profundidad de 0,1-0,2 m el contenido de arcilla en maíz y palma resultó estadísticamente mayor al de bosque y pasto, posiblemente debido a translocación de arcillas de la capa superior $(0,0-0,1 \mathrm{~m})$, donde se aprecia reducción del contenido de arcillas, por efecto de labranza o maquinaria utilizada para la cosecha de fruta, en su orden. En las profundidades de $0,3-0,4 \mathrm{~m}$ y $0,5-0,6 \mathrm{~m}$ el contenido de arcilla en palma aún siguió siendo mayor que los restantes sistemas, especialmente el bosque. La variable materia orgánica presentó un CV en profundidad que fluctuó entre $13,6 \%$ y $25,0 \%$, con diferencias estadísticas significativas en los cultivos de bosque, maíz y pasto (Tabla 3). En general los mayores contenidos de $\mathrm{MO}$ se observaron en la superficie del suelo, teniendo relación con lo expresado por Benavides et al. (2016), y sobresalió el suelo bajo cultivo de pasto; no así para los otros usos, donde la variación sucedió entre la superficie con profundidades mayores. En los pastizales es normal encontrar mayor acumulación de la $\mathrm{MO}$ en la superficie (Velázquez et al., 2008), debido a los desperdicios de pasto por el ganado que se acumulan en la superficie y por los elevados contenidos de lignina y relación $\mathrm{C} / \mathrm{N}$ que provocan una lenta mineralización de estos residuos orgánicos (Digonzelli et al., 2011).

Se encontraron diferencias estadísticas significativas para el IMO en profundidad, mas no por sistemas de uso del suelo (Tabla 3); según Vankeirsbilck (2014) valores menores a cinco, son indicativos de existencia de bajo contenido de materia orgánica y estos se observaron en las últimas profundidades de muestreo. Así, en bosque y maíz la profundidad de 0,5- 
Tabla 2. Variación en el contenido de arena, limo y arcilla en el suelo (\%) a seis profundidades, de acuerdo a la cobertura, en la Provincia de Los Ríos Ecuador.

\begin{tabular}{|c|c|c|c|c|c|c|c|c|c|c|c|c|}
\hline \multirow{2}{*}{ Profundidad } & \multicolumn{6}{|c|}{ MATERIA ORGÁNICA } & \multicolumn{6}{|c|}{ IMO } \\
\hline & B & M & $\mathrm{C}$ & $\mathbf{P}$ & PA & $\mathrm{CV}_{1}$ & B & $\mathbf{M}$ & C & $\mathbf{P}$ & PA & $\mathrm{CV}_{1}$ \\
\hline m & & & $\%$ & & & $\%$ & & & $\%$ & & & $\%$ \\
\hline $0-0,1$ & 35 & 34 & 32 & 35 & $40 \mathrm{~A}$ & $17 \mathrm{~ns}$ & 14 & 17 & $16 \mathrm{~B}$ & 16 & $14 \mathrm{C}$ & $38 \mathrm{~ns}$ \\
\hline $0,1-0,2$ & 43 & 36 & 38 & 35 & $41 \mathrm{~A}$ & $15 \mathrm{~ns}$ & $10 \mathrm{~b}$ & $17 \mathrm{a}$ & $15 \mathrm{Bab}$ & $14 \mathrm{ab}$ & $17 \mathrm{BCa}$ & $16^{*}$ \\
\hline $0,2-0,3$ & 32 & 33 & 34 & 27 & $28 \mathrm{~B}$ & $18 \mathrm{~ns}$ & 17 & 24 & $22 \mathrm{AB}$ & 19 & $32 \mathrm{AB}$ & $31 \mathrm{~ns}$ \\
\hline $0,3-0,4$ & $40 \mathrm{a}$ & $32 a b$ & $32 a b$ & $35 \mathrm{ab}$ & $25 \mathrm{Bb}$ & $17^{*}$ & $13 \mathrm{~b}$ & $26 a b$ & $30 \mathrm{ABab}$ & $20 a b$ & $38 \mathrm{Aa}$ & $30^{*}$ \\
\hline $0,4-0,5$ & 36 & 32 & 33 & 32 & $22 \mathrm{~B}$ & $19 \mathrm{~ns}$ & 18 & 30 & $33 \mathrm{~A}$ & 24 & $38 \mathrm{~A}$ & $26 \mathrm{~ns}$ \\
\hline $0,5-0,6$ & $40 \mathrm{a}$ & $36 a b$ & $32 a b$ & $38 \mathrm{ab}$ & $20 \mathrm{Bb}$ & $21^{*}$ & $14 \mathrm{~b}$ & & 34 Aab & $19 a b$ & $45 \mathrm{Aa}$ & $38^{*}$ \\
\hline CV2 (\%) & $13 \mathrm{~ns}$ & $16 \mathrm{~ns}$ & $8 \mathrm{~ns}$ & $16 \mathrm{~ns}$ & $14^{* * *}$ & & $21 \mathrm{~ns}$ & 26 & $21^{* * *}$ & $24 \mathrm{~ns}$ & $18^{* * *}$ & \\
\hline
\end{tabular}

B: bosque, M: maíz, C: cacao, P: pasto, PA: palma aceitera, $\mathrm{CV}_{1}(\%)$ : Coeficiente de variación por sistemas de uso de suelo, $\mathrm{CV}_{2}(\%)$ : Coeficiente de variación por profundidades, ns: no significativo estadísticamente, *: significativo al 5\%, **: significativo al $1 \%,{ }^{* * *}$ : significativo al $0,1 \%$, letras minúsculas: significancia entre sistemas de uso de suelo, letras mayúsculas: significancia entre profundidades.

$0,6 \mathrm{~m}$ fue estadísticamente diferente a la profundidad de $0-0,2 \mathrm{~m}$, en pasto, cacao y palma la profundidad de 0,0-0,1 m fue diferente a las demás profundidades. Los CV fluctuaron entre $16,4 \%$ y $19,9 \%$, que dan seguridad en la información. Los valores de IMO observados en superficie indican que el maíz y cacao responderán a la fertilización, mejorando la producción de los cultivos, más que en suelos de bosque, pasto y palma, donde los valores de IMO indican suelos con mayor fertilidad.

En general, en profundidad el suelo bajo bosque presentó mayor acumulación de MO que los restantes usos estudiados.
Para conocer la relación existente entre las diferentes variables evaluadas, se realizó el análisis de correlación de Pearson (Tabla 4) evidenciando que la porosidad total tiene relación negativa altamente significativa con la densidad aparente del suelo $(r=-0,5)$; es decir, si la Pt aumenta, la Da disminuye. Las principales correlaciones encontradas fueron que la Hv es altamente dependiente de la Da, Pa y Pt, las dos primeras negativas y la tercera positiva. La ADA presentó relación negativa significativa $(r=-0,57)$ con el grado de floculación y positiva $(r$ $=0,16)$ con el porcentaje de arcilla. La presencia de arcilla en el suelo, provocó efectos sobre el comportamiento de las

Tabla 3. Variación en el contenido de materia orgánica en el suelo (\%) a seis profundidades, de acuerdo a la cobertura, en la Provincia de Los Ríos Ecuador.

\begin{tabular}{|c|c|c|c|c|c|c|c|c|c|c|c|c|}
\hline \multirow{2}{*}{ Profundidad } & \multicolumn{6}{|c|}{ MATERIA ORGÁNICA } & \multicolumn{6}{|c|}{ IMO } \\
\hline & B & $\mathbf{M}$ & $\mathrm{C}$ & $\mathbf{P}$ & PA & $\mathrm{CV}_{1}$ & B & $\mathbf{M}$ & $\mathrm{C}$ & $\mathbf{P}$ & PA & $\mathrm{CV}_{1}$ \\
\hline $\mathbf{m}$ & & & $\%$ & & & $\%$ & & & $\%$ & & & $\%$ \\
\hline $0-0,1$ & $5,2 \mathrm{~A}$ & $4,9 \mathrm{~A}$ & 4,6 & $6,5 \mathrm{~A}$ & 5,6 & $29 \mathrm{~ns}$ & $0,08 \mathrm{~A}$ & $0,07 \mathrm{~A}$ & $0,07 \mathrm{~A}$ & $0,10 \mathrm{~A}$ & $0,08 \mathrm{~A}$ & $2 \mathrm{~ns}$ \\
\hline $0,1-0,2$ & $4,3 \mathrm{AB}$ & $3,5 \mathrm{AB}$ & 4,2 & $4,3 \mathrm{BC}$ & 4,6 & $37 \mathrm{~ns}$ & $0,08 \mathrm{~A}$ & $0,06 \mathrm{~A}$ & $0,05 \mathrm{AB}$ & $0,07 \mathrm{~B}$ & 0,05 B & $2 \mathrm{~ns}$ \\
\hline $0,2-0,3$ & $3,9 \mathrm{AB}$ & $3,2 \mathrm{AB}$ & 4,2 & $3,5 \mathrm{BC}$ & 4,5 & $42 \mathrm{~ns}$ & $0,06 \mathrm{AB}$ & $0,05 \mathrm{AB}$ & $0,05 \mathrm{AB}$ & 0,05 B & 0,04 BC & $26 \mathrm{~ns}$ \\
\hline $0,3-0,4$ & $3,5 \mathrm{AB}$ & $2,7 \mathrm{~B}$ & 4,1 & $3,1 \mathrm{BC}$ & 4,4 & $52 \mathrm{~ns}$ & $0,06 \mathrm{AB}$ & $0,04 \mathrm{AB}$ & $0,04 \mathrm{~B}$ & 0,05 B & $0,03 \mathrm{BC}$ & $9 \mathrm{~ns}$ \\
\hline $0,4-0,5$ & $3,3 \mathrm{~B}$ & $2,5 \mathrm{~B}$ & 4,1 & $2,8 \mathrm{C}$ & 4,3 & $59 \mathrm{~ns}$ & $0,05 \mathrm{AB}$ & $0,04 \mathrm{AB}$ & $0,04 \mathrm{~B}$ & $0,04 \mathrm{~B}$ & $0,02 \mathrm{C}$ & $1 \mathrm{~ns}$ \\
\hline $0,5-0,6$ & $2,6 \mathrm{~B}$ & $2,0 \mathrm{~B}$ & 4,0 & $2,6 \mathrm{C}$ & 4,1 & $69 \mathrm{~ns}$ & $0,04 \mathrm{~B}$ & $0,03 \mathrm{~B}$ & 0,04 B & $0,04 \mathrm{~B}$ & $0,02 \mathrm{C}$ & $8 \mathrm{~ns}$ \\
\hline CV2 (\%) & $16^{* * *}$ & $23,4^{* *}$ & $17 \mathrm{~ns}$ & $14^{* * *}$ & $25 \mathrm{~ns}$ & & $17^{*}$ & $25^{*}$ & $16,8^{* *}$ & $16^{* * *}$ & $20 * * *$ & \\
\hline
\end{tabular}

B: bosque, M: maíz, C: cacao, P: pasto, PA: palma aceitera, $\mathrm{CV}_{1}(\%)$ : Coeficiente de variación por sistemas de uso de suelo, CV 2 (\%): Coeficiente de variación por profundidades, ns: no significativo estadísticamente, *: significativo al $5 \%$, **: significativo al $1 \%$, ***: significativo al $0,1 \%$, letras minúsculas: significancia entre sistemas de uso de suelo, letras mayúsculas: significancia entre profundidades. 
TEMAS AGRARIOS - Vol. 23:(2) Julio - Diciembre 2018 (177 - 187)

Tabla 4. Correlación de Pearson entre propiedades físicas del suelo

\begin{tabular}{lccccccccccccc}
\hline Variables & CH & DA & DR & PT & PA & HV & ADA & GF & MO & IMO & ARENA & LIMO & ARCILLA \\
\hline CH & & & & & & & & & & & & & \\
DA & $-0,11$ & & & & & & & & & & & & \\
DR & $-0,01$ & 0,18 & & & & & & & & & & & \\
PT & 0,11 & $-0,50$ & 0,22 & & & & & & & & & & \\
PA & $-0,16$ & $-0,11$ & 0,33 & 0,40 & & & & & & & & & \\
HV & 0,24 & $-0,47$ & $-0,07$ & 0,32 & $-0,61$ & & & & & & & & \\
ADA & $-0,02$ & 0,09 & 0,21 & 0,05 & 0,12 & $-0,04$ & & & & & & & \\
GF & $-0,1$ & $-0,06$ & $-0,02$ & $-0,05$ & $-0,12$ & 0,04 & $-0,57$ & & & & & & \\
MO & 0,19 & $-0,03$ & $-0,42$ & $-0,16$ & $-0,23$ & 0,09 & $-0,38$ & 0,31 & & & & & \\
IMO & 0,33 & $-0,31$ & $-0,41$ & 0,12 & $-0,16$ & 0,27 & $-0,2$ & 0,10 & 0,65 & & & & \\
ARENA & 0,04 & $-0,27$ & $-0,3$ & 0,02 & $-0,06$ & 0,13 & $-0,18$ & 0,30 & 0,27 & 0,33 & & & \\
LIMO & 0,12 & $-0,07$ & $-0,2$ & 0,03 & $-0,31$ & 0,32 & $-0,05$ & 0,16 & 0,33 & 0,36 & $-0,002$ & & \\
ARCILLA & $-0,12$ & 0,24 & 0,36 & $-0,04$ & 0,27 & $-0,32$ & 0,16 & $-0,33$ & $-0,42$ & $-0,48$ & $-0,69$ & $-0,79$ & \\
\hline
\end{tabular}

$\mathrm{CH}$, conductividad hidráulica; DA, Densidad aparente del suelo; DR, Densidad real; PT, Porosidad total; PA, Porosidad de aireación; HV, Humedad volumétrica; ADA, Arcilla dispersa en agua; GF, Grado de floculación; MO, Materia orgánica; IMO, Índice de materia orgánica. Números en negrita: estadísticamente significativos.

diferentes variables evaluadas, solo no se encontraron correlaciones con la Ch y Pt.

Para profundizar en la interpretación de los resultados se realizó un análisis multivariado, evidenciándose la diferencia entre palma y los demás usos del suelo (Figura 1), las propiedades del suelo que lo diferenciaron fueron el porcentaje de arcilla y arcilla dispersa en agua, la densidad aparente y real; también se observó que los cultivos de maíz y cacao guardaron relación y los sistemas de bosque y pasto fueron semejante, diferenciándose de la palma aceitera, debido al contenido de arena, limo, $\mathrm{MO} / \mathrm{Li}+$ Arc y conductividad hidráulica.

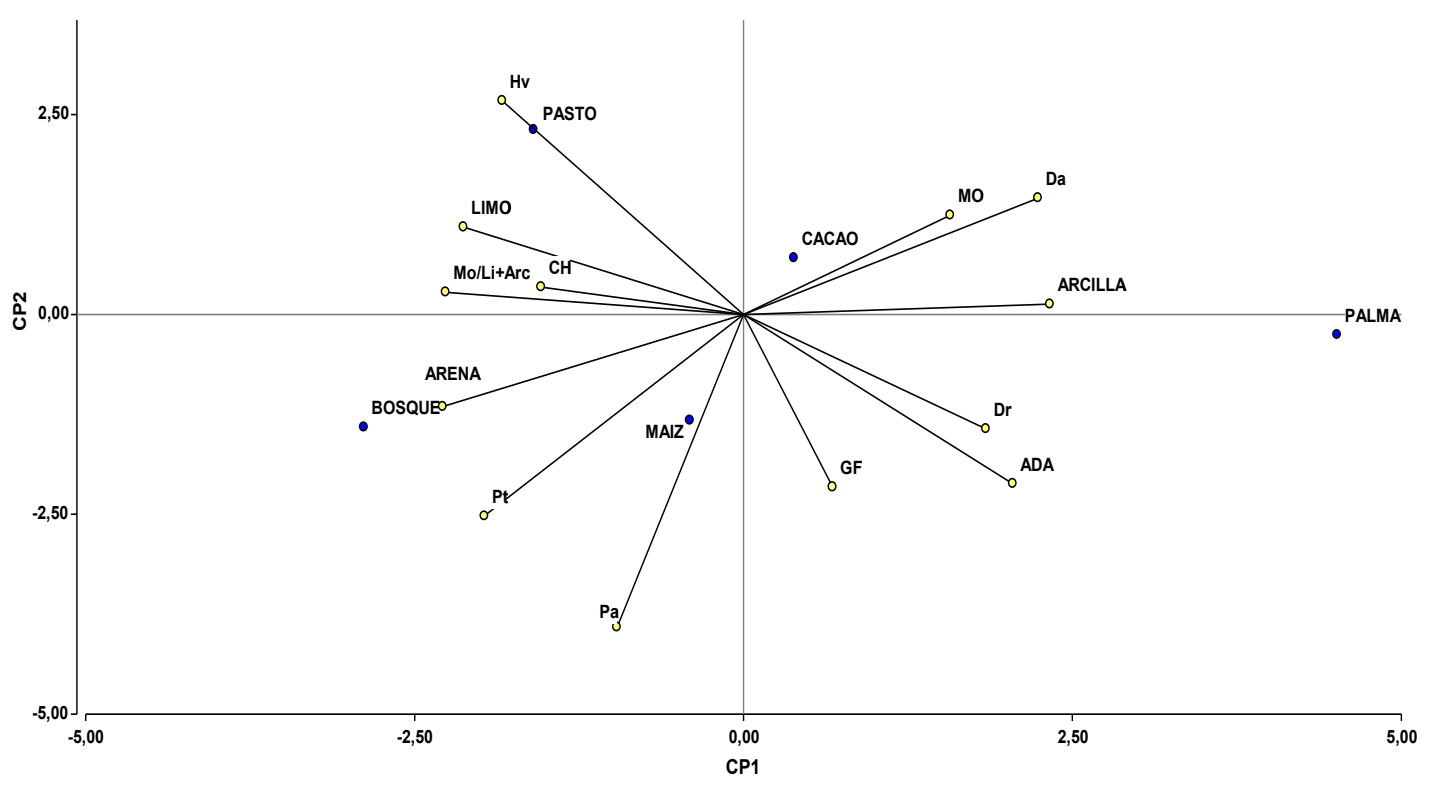

Figura 4. Análisis multivariado de las propiedades físicas de los sistemas de uso de suelo evaluados. Ch: Conductividad hidráulica, Da: densidad aparente del suelo, Dr: densidad real, Pt: porosidad total, Pa: porosidad de aireación, Hv: humedad volumétrica, ADA: Arcilla dispersa en agua, GF: grado de floculación, MO: materia orgánica, MO/Li+Arc: materia orgánica / limo+arcilla. 


\section{CONCLUSIONES}

El cambio de bosque nativo a monocultivo, modificó las características físicas del suelo, especialmente en la parte superficial. Los monocultivos de maíz, cacao, pasto y palma aceitera, aumentaron las concentraciones de arcilla en las primeras profundidades del suelo, afectando negativamente el movimiento vertical del agua.

El monocultivo de palma aceitera fue el que provocó los mayores cambios en las propiedades físicas del suelo, modificando la textura que influyó directamente sobre la $\mathrm{Da}, \mathrm{Ch}, \mathrm{ADA}$.

\section{AGRADECIMIENTOS}

Especial agradecimiento hacen referencia los autores a la Universidad Tecnológica Equinoccial, Sede Santo Domingo y la Estación Experimental Tropical Pichilingue delINIAP, porlasfacilidadesyapoyobrindado para la realización de la investigación.

\section{REFERENCIAS}

Abreu, S., Reichert, J. y Reinert, D. 2004. Escarificação mecânica e biológica para a redução da compactação em Argissolo franco-arenoso sob plantio direto. R. Bras. Ci. Solo, 28, 519- 531. https://doi.org/10.1590/ S $0100-06832004000300013$

Alonso, V., Buduba, C., Davel, M., Irrisarri, J., La Manna, L. y Puentes, C. 2007. Comparación de métodos analíticos para la determinación de materia orgánica en suelos de la región AndinoPatagónica: efectos de la vegetación y el tipo de suelo. Ciencia del Suelo, 25(1).

Alvarado, A. y Forsythe, W. 2011. Variación de la densidad aparente en órdenes de suelos de Costa Rica. Revista Agronomía Costarricense, 29(1), 85-94.
Álvarez, R. y Steinbach, H. 2009. A review of the effects of tillage systems on some soil physical properties, water content, nitrate availability and crops yield in the Argentine Pampas. Soil and Tillage Research, 104(1),1-15. https:// doi.org/10.1016/j.still.2009.02.005

Gardi, C., Angelini, M., Barceló, S., Comerma, J., Cruz Gaistardo, C., Encina Rojas, A., Jones, A., Krasilnikov, P., Mendonça Santos Brefin, M., Montanarella, L., Muñiz, O., Schad, P., Vara, M. y Vargaz, R. 2014. (eds). Atlas de suelos de américa latina y el caribe. Comisión Europea Oficina de Publicaciones de la Unión Europea, L-2995 Luxembourg. p. 50.

Barbecho, J. y Calle, J. 2012. Caracterización de la conductividad hidráulica de los suelos de la subcuenca del Río Tarqui. Tesis Ingeniería Civil. Universidad de Cuenca, Cuenca.

Bastos, M. y Oliveira, S. 2004. A dependência entre a condutividade hidráulica saturada e atributos físicos do solo. Revista Ciência Rural, 34. 963-969. https://doi.org/10.1590/ S $0103-84782004000300052$

Bravo, C., Lozano, Z., Hernández, R., Piñango, L. y Moreno, B. 2004. Efecto de diferentes especies de coberturas sobre las propiedades físicas de un suelo de sabana con siembra directa de maíz. Bioagro, 16(3).

Brissio, P. y Savini. M. 2005. Evaluación preliminar del estado decontaminación en suelos de la provincia de Neuquen donde se efectuaron actividades de explotación hidrocarburífera. Tesis Licenciado en Saneamiento y Protección Ambiental. Escuela Superior de Salud y Ambiente. Universidad Nacional de Comahue, AR.

Condé, M., Homem, B., De Almeida, O. y Santiago, A. 2012. Influência da aplicação de águas residuárias de criatórios de animais no solo: Atributos químicos e físicos. Revista Brasileira de Agropecuária Sustentável, 2(1). 
Digonzelli, A., Romero, R., Alonso, L., Ullivarri, F., Quinteros, R., Scandaliaris, J. y Fajre, S. 2011. Assessing a sustainable sugarcane production system in Tucumán, Argentina. Part 1: dynamics of sugarcane harvest residue (trash) decomposition. Revista industrial y agrícola de Tucumán, 88(1), 1-12.

Di Rienzo, J.A., F. Casanoves, M.G. Balzarini, L. Gonzalez, M. Tablada, and C.W. Robledo. 2011. InfoStat V2011. InfoStat Group, College of Agricultural Sciences, National Universidad de Córdoba, Córdoba, Argentina.

Díaz-Zorita, M. Álvarez, C. y Barraco, M. 2004. Efectos de doce años de labranzas en un Hapludol del Noroeste de Buenos Aires, Argentina. Revista Ciencia del Suelo, 22(1), 11-18.

EMBRAPA. 2011. Manual de métodos de análises de solo. (3 Ed). Rio de Janeiro: Centro Nacional de Pesquisas do Solos. 230.

Eyherabide, M., Saínz Rozas, H., Barbieri, P. y Echeverría, H. 2014. Comparación de métodosparadeterminarcarbonoorgánico en suelo. Ciencia del suelo, 32(1), 13-19.

FAO. 2000. Manual de prácticas integradas de manejo y conservación de suelos: 53. Recuperado de: https://books. google.es/books?id=-kZCpFv-W1EC\& printsec $=$ frontcover $\& d q=i s b n: 978925$ $3044177 \& \mathrm{hl}=\mathrm{es} \& \mathrm{sa}=\mathrm{X} \& \mathrm{ved}=0 \mathrm{ahUKE}$ wiOlc2Pg5fQAhUQxmMKHTQyD7Y Q6AEIHDAA\# $v=$ onepage \& $q \& f=$ false.

FAO. 2011. Guidelines for qualitative assessment of land resources and degradation. FAO. Rome, Italy. ftp://ftp.fao.org/agl/ agll/docs/misc33.pdf. [10 Junio 2017].

FAO. 2016. Propiedades físicas del suelo. Recuperado de http://www.fao. org/soils-portal/levantamientode-suelos/propiedades-del-suelo/ propiedades-fisicas/es/. [15 Junio 2017].

Ferreras, L., Toresani, S., Faggioli, V. y Galarza, C. 2015. Sensibilidad de indicadores biológicos edáficos en un Argiudol de la Región Pampeana Argentina. Revista Spanish Journal of Soil Science, 5(3).
Gabriels, D. y Lobo, D. 2011. Métodos para determinar granulometría y densidad aparente del suelo. Venesuelos, 14(1), 37-48.

Guatibonza, M., Álvarez-Herrera, J. y Sanabria, J. 2009. Distribución espacial de la conductividad hidráulica en un lote de la granja Tunguavita (Paipa, Colombia). Agronomía Colombiana, 27(2): 261.

Hillel, D. 2013. Introduction to soil physics. New York: Academic Press. 364.

Klein, V. 2008. Física do Solo. Paso Fundo, Brasil, Editora Universitaria. 212p.

Kutílek, M. y Nielsen, D. 1994. Soil hydrology: texbook for students of soil science, agriculture, forestry, geoecology, hydrology, geomorphology and other related disciplines. Catena Verlag.

Lima, J., Souza, G, y Silva, A. 2013. Distribuição espacial da matéria orgânica, grau de floculação e argila dispersa em água em área de vegetação natural em regeneração e pastagem. Revista Árvore, 37(3), 539-546. http://dx.doi.org/10.1590/ S $0100-67622013000300017$

Loss, A., Costa, E., Pereira, M. y Beutler, S. 2014. Agregação, matéria orgânica leve e carbono mineralizável em agregados do solo. La Plata, 113, 1-8.

Lozano, J., Madero, E., Tafur, H., Herrera, O. y Amézquita, E. 2005. La conductividad hidráulica del suelo estudiada en el Valle del Cauca con el nuevo indicador del USDA. Acta Agronómica, 54(3), 11-18.

Miralles, I. 2006. Calidad de Suelos en Ambientes Calizos Mediterráneos: Parque Natural de Sierra María-Los Vélez. Tesis doctoral. Departamento de Edafología y Química Agrícola. Universidad de Granada.

Muñoz, D., Ferreira. M., Arriaga, I. y López, J. 2013. Relación entre la cobertura del terreno y la degradación física y biológica De un suelo aluvial en una región semiárida. Recuperado de http://www.redalyc.org/articulo. oa? id=57328903004. [29 Junio 2017]. 
Pragana, R., Ribeiro, M., Nóbrega, J., Ribeiro Filho, M. y Costa, J. 2012. Qualidade física de Latossolos Amarelos sob plantio direto na região do Cerrado piauiense. Revista Brasileira de Ciência do Solo, 36(1), 1591-1600. https://doi.org/10.1590/ S $0100-06832012000500023$

Quiroga, A., Furano, D., Noellemeyer, E. y Peinemann, N. 2006. Barley yield response to soil organic matter and texture in the Pampas of Argentina. En: Soil \& Tillage Research, 90, 63-68. https:// doi.org/10.1016/j.still.2005.08.019

Ramírez, J., Fernandez, Y., González, P., Salazar, X., Iglesias, J. y Olivera, Y. 2015. Influencia de la fertilización en las propiedades físico-químicas de un suelo dedicado a la producción de semilla de Megathyrsus maximus. Pastos y Forrajes, 38(4), 393-402.

Rauber, L., Piccolla, C., Andrade, A., Friederichs, A., Mafra, Á., Corrêa, J. y Albuquerque, J. 2012. Physical properties and organic carbon content of a Rhodic Kandiudox fertilized with pig slurry and poultry litter. Revista Brasileira de Ciência do Solo, 36(4), 1323-1332. https://doi.org/10.1590/ S $0100-06832012000400026$

Reinert, D., Albuquerque J., Reichert J., Aita, E. y Andrada, M. 2008. Limites críticos de densidade do solo para o crescimento de raízes de plantas de cobertura em Argissolo Vermelho. Rev. Bras. Ciência Solo, 32, 1805-1816. https://doi.org/10.1590/ S $0100-06832008000500002$
Santos, L, Passos, R, Silva, L., Oliveira, P., Garcia, G. y Cecílio, R. 2010. Avaliação de alguns atributos físicos de um Latossolo Vermelho-amarelo sob diferentes coberturas vegetais. Bioscience Journal, 26, 940-947.

Silva, V. 2003. Propriedades físicas e hídricas em solos sob diferentes estados de compactação. Tese Doutorado. UniversidadeFederaldeSantaMaría, 171p.

Suquilanda, M. 2008. XI Congreso Ecuatoriano de la Ciencia del Suelo. Universidad Central del Ecuador. Recuperado de http://www.secsuelo. org/wp-content/uploads/2015/06/3.Ing.-Manuel-Suquilanda.-Suelos.pdf.

Toledo, D., Galantini, J., Ferreccio, E., Arzuaga, S., Gimenez, L. y Vázquez, S. 2013. Indicadores e índices de calidad en suelos rojos bajo sistemas naturales y cultivados. RevistaCiencia del suelo, 31(2), 201-2012.

Vankeirsbilck, M., Barraco, M. y Maekawa, M. 2014. Materia orgánica y textura en suelos de sistemas lecheros de la cuenca oeste de la provincia de Buenos Aires. Memoria técnica. EEA GeneralVillegas. 2014-2015.

Velázquez-Rodríguez, A., Flores-Román, D., Etchevers-Barra, J. y GarcíaCalderón, N. 2008. Materia orgánica en tepetate bajo cultivo de higuera y pasto, acondicionado con estiércol y fertilizante. Agrociencia, 42(1), 11-19.

Villafuerte, W. 2016. Variación en las características físicas del suelo, por efecto del uso en la estación oasis de la UTE, sede Santo Domingo de los Tsáchilas, 2015. Tesis Ingeniería Ambiental. Universidad Tecnológica Equinoccial. Santo Domingo de los Tsáchilas. 